\title{
Mechanisms of the effectiveness of poly( $\varepsilon$-caprolactone) lipid-core nanocapsules loaded with methotrexate on glioblastoma multiforme treatment
}

This article was published in the following Dove Press journal:

International Journal of Nanomedicine

\author{
Natalia Rubio Claret \\ Pereiral,* \\ Rodrigo Azevedo Loiola ${ }^{1, *}$ \\ Stephen Fernandes \\ Rodrigues' \\ Catiuscia $\mathrm{P}$ de Oliveira ${ }^{2}$ \\ Sabrina L Büttenbender ${ }^{3}$ \\ Silvia S Guterres ${ }^{2}$ \\ Adriana R Pohlmann ${ }^{3}$ \\ Sandra H Farsky' \\ 'Department of Clinical and \\ Toxicological Analysis, Faculty of \\ Pharmaceutical Sciences, University of \\ São Paulo, Sao Paulo, Brazil; '2Faculty \\ of Pharmacy, Universidade Federal \\ do Rio Grande do Sul, Porto Alegre, \\ Brazil; ${ }^{3}$ Department of Organic \\ Chemistry, Institute of Chemistry, \\ Universidade Federal do Rio Grande \\ do Sul, Porto Alegre, Brazil \\ *These authors contributed equally \\ to this work
}

\begin{abstract}
Purpose: The low penetration of drugs across the blood-brain barrier (BBB) compromises the delivery of chemotherapeutic agents to the brain parenchyma and contributes to the poor prognosis of glioblastoma multiforme (GBM). We investigated the efficacy of methotrexate-loaded lipid-core nanocapsules (MTX-LNC) administered by the oral route to treat murine GBM, its ability to cross the BBB, and the mechanisms of MTX-LNC uptake by cultured GL261 glioma and BV2 microglia cells.
\end{abstract}

Materials and methods: Female $\mathrm{C} 57 \mathrm{~B} / 6$ mice were used in intravital microscopy assays to investigate the penetrance of rhodamine B-label MTX-LNC (RhoB/MTX-LNC) in the brain after oral or IV administration, and to evaluate the BBB integrity. Intracranial implantation of GL261 cells was undertaken to induce a murine GBM model, and the effectiveness of oral MTX or MTX-LNC treatments (started on Day 10 of GBM, every 2 days for 12 days) was quantified by tumor size, body weight, and leukogram. Pharmacological blockade of endocytic pathways was done to investigate the mechanisms of MTX-LNC uptake by cultured GL261 and microglia BV2 cells by using fluorescence microscopy. The effect of MTX-LNC or MTX on GL261 and BV2 proliferation was evaluated to compare the cytotoxicity of such compounds.

Results: RhoB/MTX-LNC was detected in brain parenchyma of mice after IV or oral administration, without any damage on BBB. Oral treatment with MTX-LNC reduced tumor volume and prevented weight loss and leukopenia in comparison to MTX-treated mice. MTX-LNC uptake by GL261 is caveolae-dependent, whereas endocytosis of MTX-LNC by BV2 occurs via phagocytosis and macropinocytosis. Both MTX-LNC and MTX reduced GL261 and BV2 proliferation; however, MTX-LNC showed higher efficacy in the inhibition of glioma proliferation.

Conclusion: Together, we infer that the higher ability of MTX-LNC to cross the BBB and be captured by cancer and immune brain cells by different mechanisms is responsible for the higher efficacy of oral MTX-LNC treatment in GBM.

Keywords: glioma, nanomedicine, blood-brain barrier, microglia, endocytosis

\section{Introduction}

Glioblastoma multiforme (GBM) is the most common and aggressive malignant primary brain tumor. ${ }^{1}$ The inability of drugs to cross the blood-brain barrier (BBB) to access the tumor site is the most prominent obstacle to the effectiveness of available chemotherapy. ${ }^{2}$ For this reason, higher doses of medicines are required to achieve therapeutic concentrations in the brain, causing severe systemic toxic adverse effects. ${ }^{3}$ 
Therefore, innovative pharmacological approaches are imminently needed to provide effective GBM treatments.

Over the past few years, it has been proposed that the incorporation of medicines to nanoparticles can be an effective strategy to overcome physiological barriers, including the BBB. ${ }^{4,5}$ Based on this hypothesis, our group has previously shown that methotrexate (MTX) incorporated into poly( $\varepsilon$-caprolactone) lipid-core nanocapsules (LNC) and administered by the intraperitoneal route was more efficient than a conventional MTX solution for the treatment of a rat glioma model. ${ }^{6}$ It is noteworthy that MTX presents a narrow safety margin and low penetration across the BBB, which limits its therapeutic use for GBM treatment. ${ }^{7}$ The higher efficiency of MTX-LNC on GBM treatment is possibly associated with enhanced drug delivery at the tumor site; our group has previously shown that LNC is a technological platform suitable to carry medicines across the BBB. ${ }^{8}$

Once within the tumor site, LNC needs to be captured by surrounding cells and deliver the loaded drug to exert its therapeutic effect. These processes are highly complex and depend on physicochemical characteristics of nanoparticles and microenvironment such as the cell population at the site of therapeutic action. ${ }^{9-11}$ Taking into account the GBM microenvironment, it has been shown that surrounding cells act to favor tumor development and invasiveness. ${ }^{12,13}$ Increasing evidence has shown that infiltrated macrophages and resident microglia acquire a tumor-associated macrophages (TAMs) phenotype to facilitate tumor progression. ${ }^{12,13}$ Therefore, targeting microglia on GBM therapy may be further considered. ${ }^{14}$

Considering these abovementioned aspects, the present study was designed to elucidate two gaps of knowledge with regard to the beneficial effects of LNC on GBM treatment: 1) the ability of orally administered MTX-LNC to reach brain parenchyma and its effectiveness in the treatment of a murine GBM model; and 2) the mechanisms involved in MTX-LNC uptake by glioma and microglia cells. Data obtained herein show the ability of MTX-LNC to cross the BBB and its uptake by central nervous system (CNS) cancers and immune cells, which may be pivotal to developing higher efficacy of MTX-LNC oral treatment in GBM.

\section{Materials and methods}

\section{Animals}

C57Bl/6 female mice, age 10-14 weeks, were obtained from the breeding stock of the Pharmaceutical Sciences Faculty (University of São Paulo). All procedures were approved by the Ethics Committee of the University of São Paulo (protocol number 402), according to the Guide for the Care and Use of Laboratory Animals (NIH publication no. 85-23, revised 1996).

\section{Cell culture}

GL261, a murine glioma cell line, was cultured with Roswell Park Memorial Institute (RPMI) medium supplemented with $10 \%$ fetal bovine serum (Thermo Fisher Scientific, Waltham, MA, USA). BV2, a murine microglia cell line, was cultured with RPMI medium supplemented with $10 \%$ calf serum (Thermo Fisher Scientific). Cells were kept in a $\mathrm{CO}_{2}$ incubator at $37^{\circ} \mathrm{C}$. Both cell lines were obtained from the Banco de Células do Rio de Janeiro (RJ, Brazil).

\section{Nanocapsule preparation}

The MTX-LNC were synthesized as previously reported, ${ }^{6}$ and its features are fully described in Table 1 and Figure S1. For endocytosis and permeability studies, we used MTXLNC conjugated to rhodamine B (RhoB/MTX-LNC), as previously described. ${ }^{8}$

\section{In vivo determination of MTX-LNC penetration across BBB}

RhoB/MTX-LNC was intravenously (IV; $100 \mu \mathrm{L}$ ) or orally $(200 \mu \mathrm{L})$ administered and a craniotomy was undertaken. The exposed brain tissue was immersed in artificial cerebrospinal fluid (CSF) and pial venules (20-50 $\mu \mathrm{m}$ diameter) were visualized under fluorescence microscopy (Axioplan 2; Carl Zeiss, Göttingen, Germany). The fluorescence intensity (arbitrary units, au) inside (li) and outside (lo) the vessels were determined by the Zen ${ }^{\circledR} 2012$ (Carl-Zeiss) and it was used in the following formula: $\Delta \mathrm{I}=1-(\mathrm{Ii}-\mathrm{Io}) / \mathrm{li}$. For analyses of oral administration, raw values of lo, but not li, were used for quantification of fluorescence and it was compared with either the earlier time of treatment or animals treated with saline (autofluorescence). This procedure was based on the protocol previously described by Rodrigues et al. ${ }^{8}$ The effect of MTX-LNC on BBB permeability was assessed by measuring the extravasation of Evans Blue into brain parenchyma, as reported by Rodrigues et al and described in Supplementary materials. ${ }^{8}$

\section{Therapeutic effect of MTX-LNC on glioma growth}

The intracranial implantation of GL261 $\left(10^{5}\right.$ cells $)$ for induction of the murine GBM model was done as previously reported and the protocol is described in Supplementary materials. ${ }^{8}$ At the tenth day after glioma implantation, 
Table I Physicochemical characterization of lipid-core nanocapsules (LNC), methotrexate-loaded lipid-core nanocapsules (MTX-LNC), and rhodamine B-labeled methotrexate-loaded lipid-core nanocapsules (RhoB/MTX-LNC) using potentiometry, electrophoretic mobility, laser diffraction, dynamic light scattering, nanoparticle tracking analysis, and liquid chromatography

\begin{tabular}{|c|c|c|c|}
\hline Analysis & LNC & MTX-LNC & RhoB/MTX-LNC \\
\hline $\mathrm{pH}$ & $5.85 \pm 0.67$ & $5.01 \pm 0.43$ & $5.03 \pm 0.27$ \\
\hline Zeta potential $(\mathrm{mV})$ & $-10.8 \pm 5.5$ & $-9.4 \pm 2.1$ & $-8.9 \pm 1.7$ \\
\hline $\mathrm{D}[4,3](\mathrm{nm})$ & $20 I \pm 8$ & $214 \pm 18$ & $236 \pm 88$ \\
\hline SPAN & $1.8 \pm 0.1$ & $1.9 \pm 0.1$ & $1.8 \pm 0.2$ \\
\hline$S A\left(m^{2} g^{-1}\right)$ & $41.6 \pm 1.7$ & $40 \pm 2.1$ & $39.1 \pm 13.4$ \\
\hline $\mathrm{D}_{\mathrm{z} \text {-ave }}(\mathrm{nm})$ & $187 \pm 7$ & $192 \pm 6$ & $189 \pm 8$ \\
\hline PDI & $0.1 \pm 0$ & $0.1 \pm 0$ & $0.1 \pm 0$ \\
\hline$D_{h}(n m)$ & $190 \pm 10$ & $216 \pm 6$ & $209 \pm 4$ \\
\hline D50 (nm) & $182 \pm 10$ & $206 \pm 6$ & $199 \pm 4$ \\
\hline D90 (nm) & $263 \pm 10$ & $295 \pm 11$ & $294 \pm 17$ \\
\hline PND (particles $\mathrm{mL}^{-1}$ ) & $(5.34 \pm 0.37) \times 10^{-12}$ & $(4.90 \pm 0.36) \times 10^{-12}$ & $(5.06 \pm 0.09) \times 10^{-12}$ \\
\hline MTX (mg mL $\left.L^{-1}\right)$ & - & $0.251 \pm 0.014$ & $0.252 \pm 0.008$ \\
\hline $\mathrm{EE}(\%)$ & - & $46.65 \pm 5.68$ & $44.48 \pm 6.38$ \\
\hline
\end{tabular}

Notes: SPAN $=\left(d_{0,}-d_{0,}\right) / d_{05}$, polydispersity calculated using laser diffraction data $\left(d_{0,}, d_{0.5}\right.$, and $d_{0,9}$ are the diameters at percentiles 10,50 , and 90 under the size distribution curve); $\left[\right.$ [4,3], volume-weighted mean diameter obtained by laser diffraction; $D_{z \text {-ave, }}$-average hydrodynamic diameter obtained by dynamic light scattering; $D_{h}$, hydrodynamic mean diameter obtained by nanoparticle tracking analysis; D50, diameter at percentile 50 under the size distribution curve obtained by nanoparticle tracking analysis; D 90 , diameter at percentile 90 under the size distribution curve obtained by nanoparticle tracking analysis.

Abbreviations: SA, surface area obtained by laser diffraction (density of particles = I); PDI, polydispersity index (relative variance); PND, particle number density obtained by nanoparticle tracking analysis; $E E$, encapsulation efficiency.

mice were treated with MTX-LNC $(250 \mu \mathrm{g} / \mathrm{mL})$, MTX $(250 \mu \mathrm{g} / \mathrm{mL})$, or saline solution, administered by gavage $(100 \mu \mathrm{L})$ every 2 days. The treatment was continued until the 20th day after glioma implantation; 2 days later, animals were anesthetized (ketamine/xylazine, $80: 8 \mathrm{mg} / \mathrm{kg}$ ) and euthanized. Brain samples were cut ( $2 \mathrm{~mm}$ coronal sections) and sections were digitized using a scanner (Epson Stylus TX235W; Manaus, Brazil). The total hemispheric and tumor areas of each brain section were quantified by ImageJ (version $1.47 \mathrm{v}$; NIH, Bethesda, MD, USA). The tumor volume was expressed as the percentage of the ipsilateral hemisphere. For leukogram, blood collected from the inferior vena cava was diluted with Turk's liquid and total leukocytes were counted by using a hemocytometer. The food intake ( $\mathrm{g}$ ) and the body mass (g) were measured just before glioma implantation (basal) and every 48 hours until Day 22.

\section{Mechanisms of MTX-LNC uptake}

GL261 and BV2 were incubated (for 60 minutes at $37^{\circ} \mathrm{C}$ ) with Hank's balanced salt solution (HBSS) containing RhoB/MTX-LNC (5.5 $\mu \mathrm{mol} / \mathrm{L})$, under conditions determined in preliminary studies (Supplementary materials). The nucleus was labeled with 4', 6-diamidino-2-phenylindole (DAPI; Sigma-Aldrich, St. Louis, MO, USA). To investigate the mechanisms involved in MTX-LNC uptake, cells were pre-incubated ( 1 hour, $\left.37^{\circ} \mathrm{C}\right)$ with inhibitors of phagocytosis (cytochalasin D [Cyto D] $10 \mu \mathrm{mol} / \mathrm{L}$ ), clathrin-mediated endocytosis (high sucrose concentration
[Suc] $0.5 \mathrm{~mol} / \mathrm{L}$ ), caveolae-mediated endocytosis (methyl$\beta$-cyclodextrin $[\mathrm{M} \beta \mathrm{C}] 10 \mu \mathrm{mol} / \mathrm{L})$, or macropinocytosis (amiloride [Amil] $1 \mathrm{mmol} / \mathrm{L}$ ) (all from Sigma-Aldrich). The concentration of Amil used was chosen in accordance with a previous report, whereas the concentrations of other blockers were selected on the basis of preliminary experiments (Figure S2). ${ }^{15}$ In order to inhibit the cell metabolism and energy-dependent endocytosis, cells were incubated with RhoB/MTX-LNC at $4^{\circ} \mathrm{C}$ for 1 hour, or pre-incubated for 1 hour at $37^{\circ} \mathrm{C}$ with the ATP-inhibitor sodium azide (Azide; $15 \mathrm{mmol} / \mathrm{L}$ ). Images were acquired by a fluorescence microscope (AxioVision ${ }^{\circledR}$; Carl-Zeiss) and the fluorescence intensity (au) was determined by using Zen 2012 (Carl-Zeiss). The results represent the percentage of RhoB/MTX-LNC uptake in comparison with cells that were incubated only with HBSS (negative control).

\section{In vitro cytotoxicity of MTX-LNC and MTX}

The cytotoxicity of MTX-LNC and MTX on GL261 and BV2 was evaluated by a proliferation assay. Briefly, BV2 or GL261 ( $5 \times 10^{3}$ cells/well) were seeded on a 98-well plate. Cells were treated (48 hours) with medium containing a serial logarithmic dilution of MTX or MTX-LNC (0.01-100 $\mu \mathrm{mol} / \mathrm{L})$. After treatments, cell monolayers were fixed for 60 minutes at $4{ }^{\circ} \mathrm{C}$ with $10 \%$ (wt/vol) trichloroacetic acid solution and then stained for 10 minutes at room temperature with $0.4 \%$ (wt/vol) sulforhodamine B (SRB) solution. Afterward, 
the excess of dye was removed by washing repeatedly with $1 \%$ (vol/vol) acetic acid. The protein-bound SRB was dissolved in $100 \mathrm{mmol} / \mathrm{L}$ Tris base solution, and absorbance was measured at $515 \mathrm{~nm}$ using a microplate reader (Synergy H1; Biotek, Winooski, VT, USA). The sigmoidal dose-response curve, which shows the response as a function of the logarithm of concentration, was calculated by using GraphPad 4.0. The effectiveness of treatments was compared by analyzing $\mathrm{IC}_{50}$.

\section{Statistical analyses}

All data were analyzed by one-way analysis of variance (ANOVA), with post hoc comparisons using the Tukey test. In all cases, a $P$-value $<0.05$ was taken as indicating statistical significance.

\section{Results and Discussion MTX-LNC crosses the BBB after intravenous or oral administration and displays high therapeutic effectiveness on GBM treatment}

The higher effectiveness of MTX-LNC in treating GBM impelled us to investigate its ability to cross the BBB.
An intravital microscopy study revealed the presence of RhoB-MTX-LNC in the tissue surrounding pial vessels 30 minutes after an IV injection $(250 \mu \mathrm{g} / \mathrm{mL}$ solution, $100 \mu \mathrm{L}$; Figure 1A), even in the absence of tumor development. Similar results were observed following oral administration of RhoB-MTX-LNC (250 $\mu \mathrm{g} / \mathrm{mL}$ solution, $200 \mu \mathrm{L}$; Figure 1B). The effect was not due to enhanced BBB permeability, as the integrity of the BBB was not altered after MTX-LNC injection (Figure 1C). Data obtained herein were similar to those observed previously by our group using LNC loaded with indomethacin and indomethacin ethyl ester, confirming the ability of LNC to penetrate brain parenchyma, independently of the loading drug. ${ }^{8}$ It is important to mention that we previously tested the in vitro chemical stability of rhodamine binding to LNC in simulated fluids, such as gastric and intestinal secretions and plasma, and strong evidences support that the fluorescence emission observed by intravital microscopy is a consequence of the labeled LNC crossing the BBB. ${ }^{8}$

Based on such observations, we tested the ability of MTX-LNC administered via the oral route to prevent tumor growth in a murine GBM model. Herein, we applied a different protocol of treatment from that reported by Figueró et al, ${ }^{7}$
A

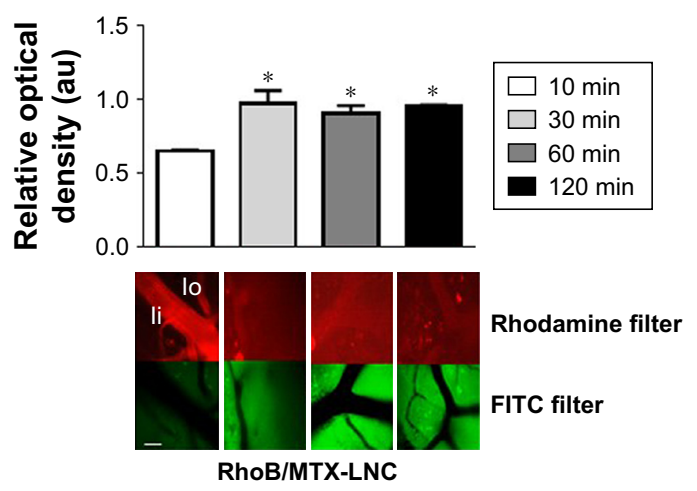

B

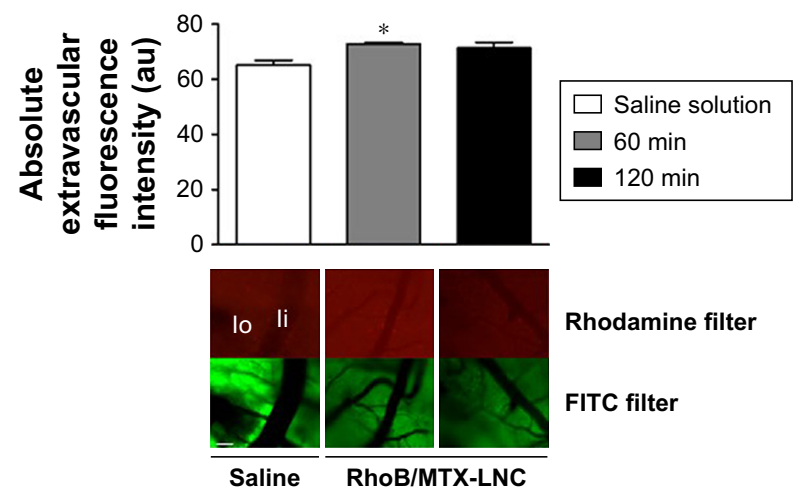

C

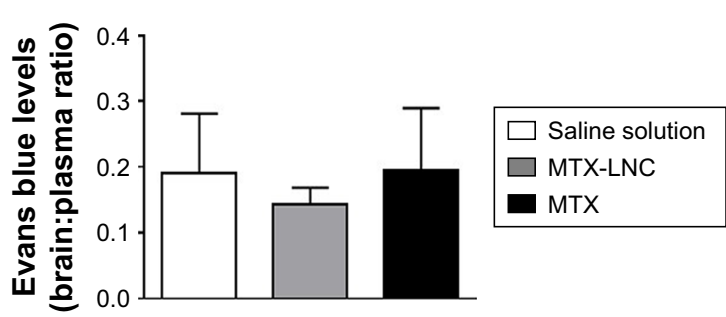

Figure I Penetration of MTX-LNC across the BBB.

Notes: Representative images and bar graphs showing intravascular (li) and extravascular (lo) optical density measurements of cerebral microvessels of mice (A) intravenously (10,30,60, or 120 minutes before visualization) or (B) orally (60 or 120 minutes before visualization) treated with RhoB/MTX-LNC. (C) Bar graphs showing BBB permeability of mice treated with saline solution, MTX-LNC, or MTX via the intravenous route. BBB permeability was evaluated by measuring Evans blue extravasation into the brain; values represent an association between the Evans blue amount in plasma and in the perfused brain. The results are expressed as mean \pm SEM, $n=3-5$ per group. Data were analyzed by one-way ANOVA, $* P<0.05$ vs control. White bar: $20 \mu \mathrm{m}$.

Abbreviations: au, arbitrary unit; FITC, fluorescein isothiocyanate; RhoB/MTX-LNC, rhodamine B-labeled methotrexate-loaded lipid-core nanocapsules; MTX, methotrexate; BBB, blood-brain barrier; SEM, standard error of the mean; ANOVA, analysis of variance; lo, outside; li, inside. 
employing half the number of doses administered on alternate days by the oral route. Following this treatment regimen, we observed that the glioma volume was significantly lower in mice treated with MTX-LNC $(250 \mu \mathrm{g} / \mathrm{mL}$ solution, $100 \mu \mathrm{L})$ in comparison to those treated with saline or MTX (250 $\mu \mathrm{g} / \mathrm{mL}$ solution, $100 \mu \mathrm{L}$; Figure 2A). All images of tumors obtained are shown in the Figure S3. Moreover, MTX-LNC prevented weight loss (Figure 2B), confirming the effectiveness of MTX-LNC on GBM treatment. Treatment with MTX may lead to profound suppression of bone marrow, with consequent immunosuppression. ${ }^{15,16}$ Indeed, the number of leukocytes in the blood of MTX-treated mice was lower than that observed in saline- or MTX-LNC-treated mice (Figure 2C), indicating that MTX-LNC lacks some systemic side effect - in particular, myelosuppression. The absence of toxicity is possibly due to the preferential delivery of MTX-LNC to the damaged brain and lower levels of the drug in healthy tissues. Although such an aspect represents an advantage in comparison to current GBM therapy, further experiments must be carried out to evaluate the toxicity of MTX-LNC on bone marrow.

\section{MTX-LNC uptake by glioma and microglial cells is mediated by different mechanisms}

Nowadays, understanding the process of nanoparticle uptake and intracellular trafficking in different cells is a challenge to the nanotechnology field, as different intracellular signaling and toxicity may be induced on the basis of cell-uptake mechanisms. Therefore, the knowledge of how nanoparticles reach cells is fundamental to design and propose efficient therapeutic approaches. ${ }^{11}$ Once MTX-LNC reaches the tumor site, the drug needs to be captured by the glioma and surrounding cells to achieve its pharmacological effect. Therefore, we investigated the mechanisms of MTX-LNC (5.5 $\mu \mathrm{mol} / \mathrm{L})$ uptake by GL261 glioma and BV2 microglial cells. The latter was employed in consideration of recent descriptions showing the positive actions of microglia on GBM development. ${ }^{12,13}$ We employed well-recognized pharmacological approaches to block the main pathways of nanoparticle cell uptake. Our data demonstrated that pretreatment with sodium azide or incubation of cells at $4^{\circ} \mathrm{C}$ impaired the endocytosis of MTX-LNC $(5.5 \mu \mathrm{mol} / \mathrm{L})$ by

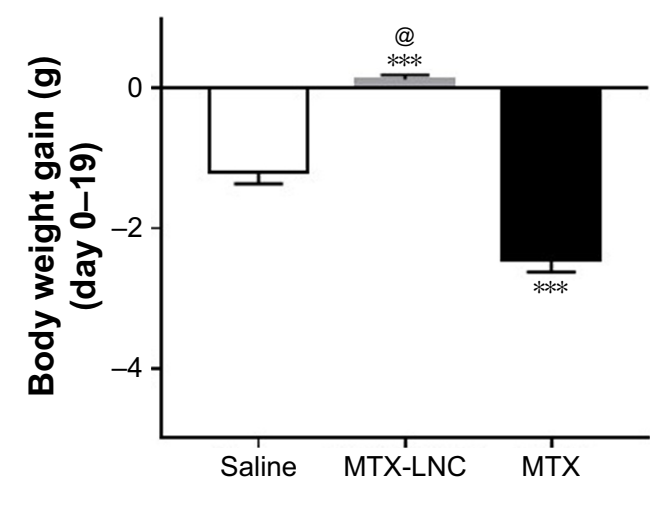

\section{B}
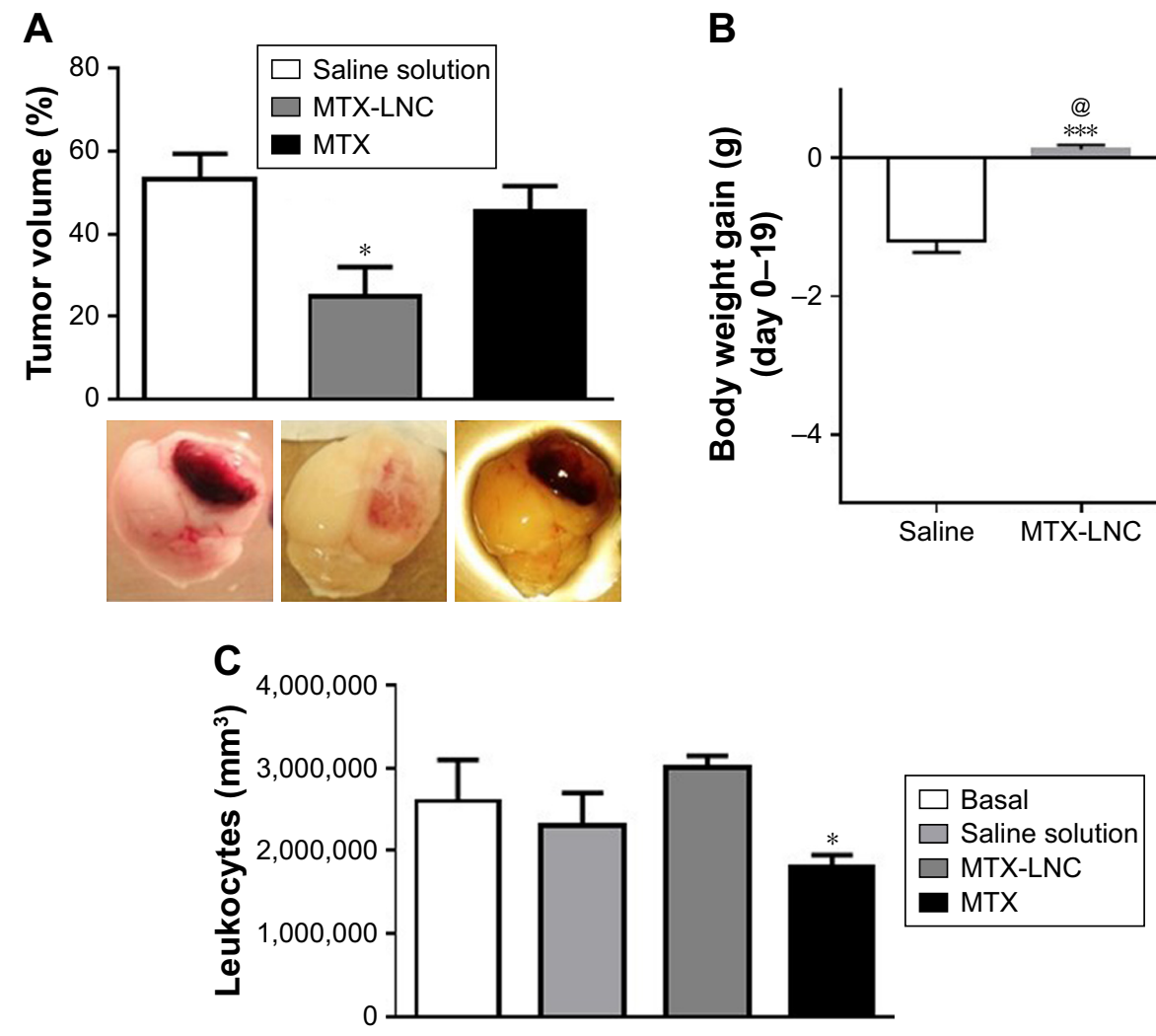

Figure 2 Effectiveness of MTX-LNC on GBM treatment.

Notes: (A) Representative images of brain and bar graphs showing tumor volume (\% compared to the ipsilateral hemisphere) of glioblastoma-carrying mice treated with saline solution, MTX-LNC, or MTX, on alternate days, by the oral route. Weight loss (g) (B) and leukogram (C) of glioblastoma-carrying mice treated with saline solution, MTX-LNC, and MTX. The results are expressed as mean \pm SEM, $n=3-5$ per group. Data were analyzed by one-way ANOVA. $* P<0.05$ vs control; *** $P<0.0$ I vs control; $@ P<0.05$ vs $M T X$. Abbreviations: MTX-LNC, methotrexate-loaded lipid-core nanocapsules; MTX, methotrexate; GBM, glioblastoma multiforme; SEM, standard error of the mean; ANOVA, analysis of variance. 


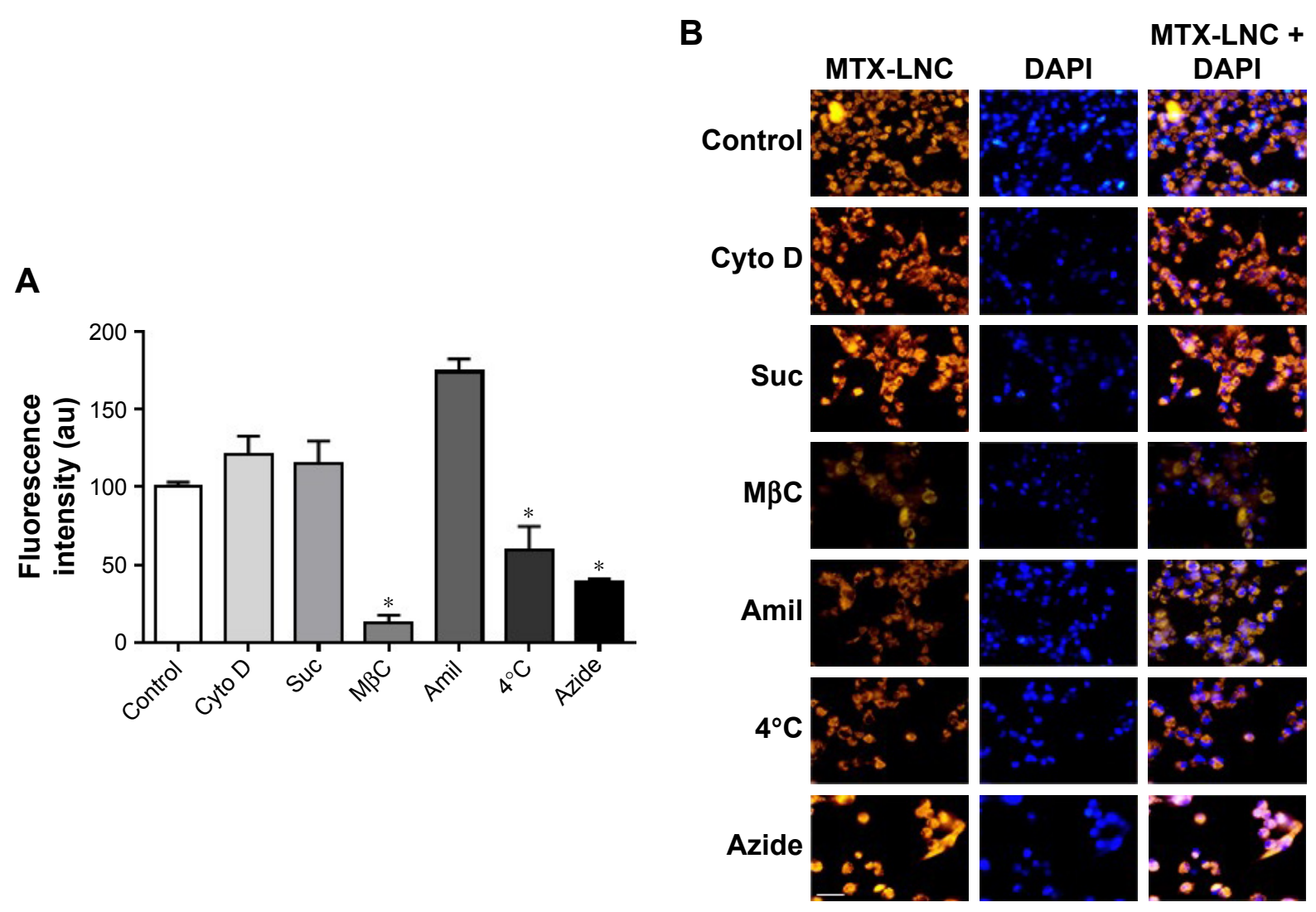

Figure 3 Mechanisms of MTX-LNC uptake by GL26I glioma cells.

Notes: (A) Bar graphs and (B) representative images of RhoB/MTX-LNC endocytosis by GL26I in the absence (control, $37^{\circ} \mathrm{C}$ ) or presence of blockers Cyto D (I0 $\mu$ mol/L), Suc $(0.5 \mathrm{~mol} / \mathrm{L})$, Amil $(\mathrm{I} \mathrm{mmol} / \mathrm{L}), \mathrm{M} \beta \mathrm{C}(5 \mu \mathrm{mol} / \mathrm{L})$, azide $(15 \mathrm{mmol} / \mathrm{L})$, or incubation at $4^{\circ} \mathrm{C}$. The results are expressed as mean $\pm \mathrm{SEM}$, $\mathrm{n}=4-6$. Data were analyzed by oneway ANOVA, $* P<0.05$ vs control. White bar: $20 \mu \mathrm{m}$.

Abbreviations: au, arbitrary unit; DAPI, 4',6-diamidino-2-phenylindole; MTX-LNC, methotrexate-loaded lipid-core nanocapsules; RhoB/MTX-LNC, rhodamine B-labeled MTX-LNC; SEM, standard error of the mean; ANOVA, analysis of variance.

both cell lines (Figures 3 and 4), showing that MTX-LNC uptake is energy dependent. In the glioma lineage GL261, MTX-LNC uptake was preferentially mediated by caveolaedependent endocytosis, once pretreatment with cyclodextrin impaired the MTX-LNC uptake by GL261 cells (Figure 3). Caveolae exist on cell surfaces as caveolin-coated invaginations, and caveolae-dependent endocytosis is involved in pivotal cell metabolism processes. ${ }^{17}$ Caveolae contains a high concentration of signaling molecules, working as a transducing center in the cell. ${ }^{17}$ Moreover, particles entering the cell by caveolae-dependent endocytosis can escape lysosomal degradation, which is interesting for the delivery of degradable materials and drugs with acid-triggered release characteristics. ${ }^{17}$ For such reasons and in association with the high expression of caveolae in solid tumor cells, we propose MTX-LNC as an alternative approach to drug delivery for cancer treatment. ${ }^{18}$

Differently, the MTX-LNC uptake by the murine microglial cell line BV2 was mediated by phagocytosis and macropinocytosis, once pretreatment with Amil or Cyto D impaired MTX-LNC endocytosis (Figure 4). Macropinocytosis is a dynamic endocytic process, wherein large membrane extensions or ruffles are formed as a result of cytoskeleton rearrangement. After particle engulfment, ruffles fuse back onto the plasma membrane, creating a large vesicle, and huge molecules are engulfed regardless of the presence of their specific receptors. ${ }^{19}$ Conversely, phagocytosis is a specific endocytic process, depending on the recognition of the particles by cell receptors on phagosomes. ${ }^{20}$ Similar to macropinocytosis, phagocytosis primarily occurs in immune cells known as professional phagocytes, including microglia. Indeed, it was shown that phagocytosis and macropinocytosis are involved in silica nanoparticle uptake in murine microglial cells and were subsequently found in the endoplasmatic reticulum and lysosomes. ${ }^{21}$ Moreover, the phagocytosis of nanoparticles by macrophages has driven the development of carriers to target drugs toward immune phagocytic cells. ${ }^{22}$ The results obtained in this study clearly indicated different mechanisms of MTX-LNC uptake by cancer and immune cells in the brain. The ability of both cells to capture MTX delivered at a higher concentration at the site of the GBM may contribute to the improved efficacy of LNC treatments. 


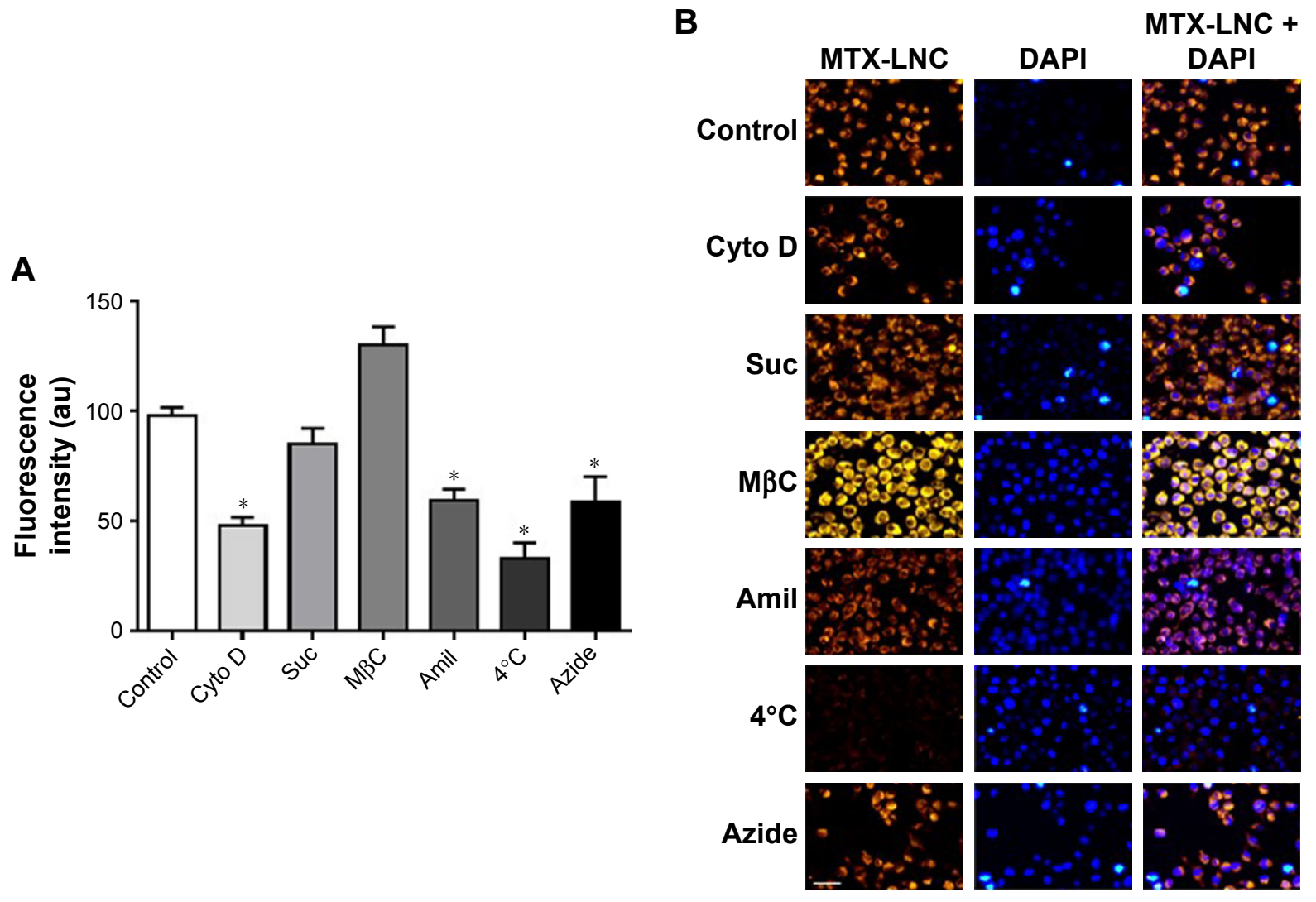

B

Figure 4 Mechanisms of MTX-LNC endocytosis by BV2 microglial cells.

Notes: (A) Bar graphs and (B) representative images of RhoB/MTX-LNC uptake by BV2 in the absence (control, $\left.37^{\circ} \mathrm{C}\right)$ or presence of blockers Cyto $\mathrm{D}(\mathrm{I} 0 \mu \mathrm{mol} / \mathrm{L})$, Suc $(0.5$ $\mathrm{mol} / \mathrm{L})$, Amil $(\mathrm{I} \mathrm{mmol} / \mathrm{L}), \mathrm{M} \beta \mathrm{C}(5 \mu \mathrm{mol} / \mathrm{L})$, azide $(15 \mathrm{mmol} / \mathrm{L})$ or incubation at $4^{\circ} \mathrm{C}$. The results are expressed as mean $\pm \mathrm{SEM}, \mathrm{n}=4-6$. Data were analyzed by one-way ANOVA, $* P<0.05$ vs control. White bar: $20 \mu \mathrm{m}$.

Abbreviations: au, arbitrary unit; DAPI, 4',6-diamidino-2-phenylindole; MTX-LNC, methotrexate-loaded lipid-core nanocapsules; RhoB/MTX-LNC, rhodamine B-labeled MTX-LNC; SEM, standard error of the mean; ANOVA, analysis of variance.

\section{MTX-LNC and MTX reduce proliferation of glioma and microglial cells}

The in vitro toxicity of MTX-LNC or MTX on GL261 and BV2 was evaluated by a proliferation assay. Data obtained herein showed marked reduction in cell proliferation that was induced by both compounds (Figure 5). It is noteworthy that MTX-LNC showed higher efficacy in the inhibition of glioma proliferation, once values of $\mathrm{IC}_{50}$ of nanocapsules on GL261 $\left(0.21 \pm 0.004 \mu \mathrm{mol} / \mathrm{L}^{*} P<0.05\right.$; Figure 5A) were significantly lower in comparison to those of BV2 $(1.03 \pm 0.025 \mu \mathrm{mol} / \mathrm{L}$;
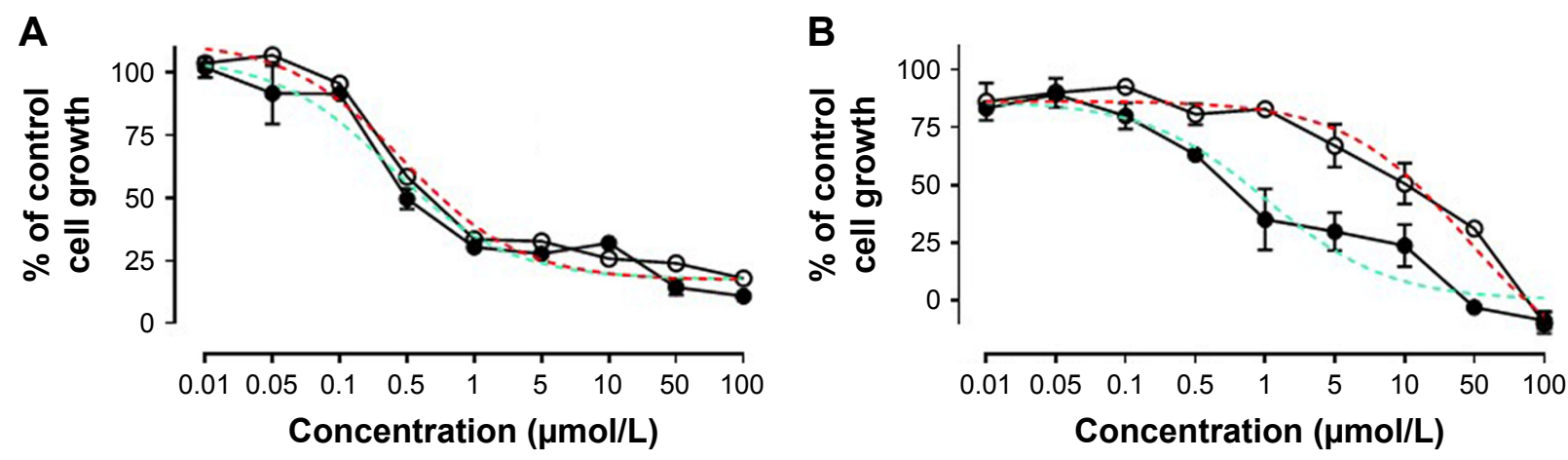

MTX-LNC $\vartheta$ MTX

Figure 5 Effect of MTX-LNC and MTX on GL26I and BV2 proliferation.

Notes: Line graphs showing the effect of MTX-LNC or MTX (0.0I-100 $\mu \mathrm{mol} / \mathrm{L})$ on GL26I (A) or BV2 (B) proliferation. The effect of MTX-LNC or MTX on proliferation is presented as the percentage of cell growth in absence of treatment. Sigmoidal dose-response curves for MTX-LNC and MTX are represented as green and red dotted lines, respectively. Data represent mean \pm SEM of three independent experiments conducted in duplicate.

Abbreviations: MTX-LNC, methotrexate-loaded lipid-core nanocapsules; MTX, methotrexate; SEM, standard error of the mean. 
Figure 5B). Although several parameters must be considered to correlate our in vitro and in vivo results, a higher effectiveness of MTX-LNC on GBM treatment might be related to the ability of nanocapsules in crossing the BBB and enhanced antiproliferative activity on glioma cells.

\section{Conclusion}

The present study clearly shows the higher therapeutic efficacy of MTX-LNC in relation to MTX on GBM treatment. We suggest that oral low doses of MTX-LNC are a safer and effective alternative to the current expensive and invasive intravenous high-dose MTX regimens. The beneficial effect of MTX-LNC may be due, at least in part, by the improved ability of LNC-loaded drugs to cross the BBB and the efficient MTX-LNC uptake by cancer and immune cells in the brain. Taken together, our results highlight the potential application of LNC-loaded drugs for the treatment of GBM and other CNS diseases. Moreover, we propose that combined LNC therapies carrying drugs to halt glioblastoma cell proliferation and microglia functions would bring high effectiveness to pharmacological approaches.

\section{Acknowledgments}

The authors thank Fundação de Amparo à Pesquisa do Estado de São Paulo (S.H.P.F. FAPESP - grant no. 2014/07328-4) and Fundação de Amparo à Pesquisa do Estado de Rio Grande do Sul (FAPERGS) for financial support to this study; Conselho Nacional de Desenvolvimento Científico e Tecnológico (CNPq) for Master fellow support to Natália Rubio Claret Pereira and also for financial support to this study (Universal 470895/2011-0); and Coordenação de Aperfeiçoamento de Pessoal de Nível Superior (CAPES) for the post-doctoral fellowship to Rodrigo Azevedo Loiola. Stephen Fernandes Rodrigues was a recipient of a postdoctoral fellowship from FAPESP (2011/02438-8). Adriana R Pohlmann, Silvia S Guterres, and Sandra H Farsky are researcher fellows of CNPq. We are grateful to Willian Prado, MSc for the synthesis of rhodamine B-LNC conjugates.

\section{Author contributions}

NRCP conducted in vivo assays; RAL conducted in vitro assays and contributed to manuscript writing; SFR undertook intravital microscopy experiments; CPO and SLB prepared and analyzed methotrexate-loaded lipid-core nanocapsules (MTX-LNC); SSG and ARP designed and developed MTXLNC; SHF conceived this study and wrote the manuscript. All authors contributed substantially to data acquisition/ analysis and drafting and/or critically revising the paper, and agree to be accountable for all aspects of the work.

\section{Disclosure}

The authors report no conflicts of interest in this work.

\section{References}

1. McFaline-Figueroa JR, Lee EQ. Brain tumors. Am J Med. Epub 2018 Jan 22. pii: S0002-9343(18)30031-7.

2. Shiekh FA. Blood-brain barrier: a real obstacle for therapeutics. Int $J$ Nanomedicine. 2012;7:4065-4066.

3. Arnone GD, Bhimani AD, Aguilar T, Mehta AI. Localized targeted antiangiogenic drug delivery for glioblastoma. J Neurooncol. 2018; 137(2):223-231.

4. Bernardi A, Braganhol E, Jäger E, et al. Indomethacin-loaded nanocapsules treatment reduces in vivo glioblastoma growth in a rat glioma model. Cancer Lett. 2009;281(1):53-63.

5. Kim CS, Mout R, Zhao Y, et al. Co-delivery of protein and small molecule therapeutics using nanoparticle-stabilized nanocapsules. Biconjug Chem. 2015;26(5):950-954.

6. Figueiró F, de Oliveira CP, Rockenbach L, et al. Pharmacological improvement and preclinical evaluation of methotrexate-loaded lipidcore nanocapsules in a glioblastoma model. J Biomed Nanotechnol. 2015; 11(10):1808-1818.

7. Abolmaali SS, Tamaddone AM, Dinarvand R. A review of therapeutic challenges and achievements of methotrexate delivery systems for treatment of cancer and rheumatoid arthritis. Cancer Chemother Pharmacol. 2013;71(5):1115-1130.

8. Rodrigues SF, Fiel LA, Shimada AL, et al. Lipid-core nanocapsules act as a drug shuttle through the blood-brain barrier and reduce glioblastoma after intravenous or oral administration. J Biomed Nanotechnol. 2016;12(5):986-1000.

9. Ma N, Ma C, Li C, et al. Influence of nanoparticle shape, size, and surface functionalization on cellular uptake. J Nanosci Nanotechnol. 2013; 13(10):6485-6498.

10. Hoshyar N, Gray S, Han H, Bao G. The effect of nanoparticle size on in vivo pharmacokinetics and cellular interaction. Nanomedicine (Lond). 2016;11(6):673-692.

11. Behzadi S, Serpooshan V, Tao W, et al. Cellular uptake of nanoparticles: journey inside the cell. Chem Soc Rev. 2017;46(14):4218-4244.

12. Roos A, Ding Z, Loftus JC, Tran NL. Molecular and microenvironmental determinants of glioma stem-like cell survival and invasion. Front Oncol. 2017;7:120.

13. Sorensen MD, Dahlrot RH, Boldt HB, Hansen S, Kristensen BW. Tumour-associated microglia/macrophages predict poor prognosis in high-grade gliomas and correlate with an aggressive tumour subtype. Neuropathol Appl Neurobiol. 2017;44(2):185-206.

14. Poon CC, Sarkar S, Yong VW, Kelly JJP. Glioblastoma-associated microglia and macrophages: targets for therapies to improve prognosis. Brain. 2017;140(6):1548-1560.

15. Koivusalo M, Welch C, Hayashi H, et al. Amiloride inhibits micropinocytosis by lowering submembranous $\mathrm{pH}$ and preventing Rac1 and Cdc42 signalling. J Cell Biol. 2010;188(4):547-563.

16. Sosin M, Handa S. Low dose methotrexate, and bone marrow suppression. BMJ. 2003;326(7383):266-267.

17. Parton RG, del Pozo MA. Caveolae as plasma membrane sensors, protectors and organizers. Nat Rev Mol Cell Biol. 2013;14(2):98-112.

18. Carver LA, Schnitzer JE. Caveolae: mining little caves for new cancer targets. Nat Rev Cancer. 2003;3:571-581.

19. Lim JP, Gleeson PA. Macropinocytosis: an endocytic pathway for internalizing large gulps. Immunol Cell Biol. 2011;89(8):836-843.

20. Gordon S. Phagocytosis: an immunobiologic process. Immunity. 2016; 44(3):463-475.

21. Zielinski J, Möller AM, Frenz M, Mevissen M. Evaluation of endocytosis of silica particles used in biodegradable implants in the brain. Nanomedicine. 2016;12(6):1603-1613.

22. Costa A, Sarmento B, Seabra V. Targeted drug delivery systems for lung macrophages. Curr Drug Targets. 2015;16(14):1565-1581. 


\section{Supplementary materials Blood-brain barrier (BBB) integrity}

In order to investigate the effects of MTX-LNC on BBB permeability, we evaluated the effect of treatments in the extravasation of Evans Blue (EB) (Sigma-Aldrich; St. Louis, MO, USA) into brain parenchyma. Briefly, EB solution ( $2 \%$ ) was injected $(4 \mathrm{~mL} / \mathrm{kg}$ ) into the femoral vein of anesthetized mice 5 minutes after IV treatment with MTX-LNC, MTX, or saline solution. Twenty-four hours later, $0.4 \mathrm{~mL}$ blood was collected and mice were transcardially perfused with PBS for 5 minutes. The brain was removed and divided into two hemispheres, and each one was homogenized and sonicated in $1 \mathrm{~mL}$ of $50 \%$ trichloroacetic acid (Sigma-Aldrich) and centrifuged (10,000 rpm, 20 minutes). The supernatant was diluted with ethanol, and EB concentrations in brain and plasma were determined by using a fluorescence spectrophotometer (FLUOstar Optima microplate reader; BMG Labtech, Inc., Ortenberg, Germany). The BBB permeability was determined by dividing cerebral EB concentration ( $\mu \mathrm{g} / \mathrm{g}$ brain weight) by the plasma EB concentration $(\mu \mathrm{g} / \mathrm{g})$.

\section{Intracranial injection of glioma cells}

The intracranial implantation of GL261 ( $10^{5}$ cells) was undertaken following the protocol previously described by Rodrigues et al. ${ }^{8}$ The entire procedure was carried out under sterile operating room conditions. Briefly, mice were anesthetized and affixed in a prone position using an acrylic stereotactic head frame. A vertical incision was made in the skin overlying the skull, exposing the coronal suture and bregma. A cranial window $\left(2 \mathrm{~mm}^{2}\right)$ was opened using a mini drill starting $1 \mathrm{~mm}$ to the right of the midline sagittal suture and $1 \mathrm{~mm}$ posterior to the coronal suture. A 26-gauge needle was inserted at a position $2.7 \mathrm{~mm}$ right of the midline sagittal suture, $0.5 \mathrm{~mm}$ posterior to the coronal suture, and at a depth of $2.4 \mathrm{~mm}$ from the surface of the brain. A cell culture solution $\left(2 \mu \mathrm{L} ; 5 \times 10^{7}\right.$ cells $\left./ \mathrm{mL}\right)$ was injected over 8 minutes with an automated microinjection system attached to a fully motorized stereotaxic system (Neurostar, Sindelfingen, Germany). Then, the needle was slowly removed, the original cranial bone was returned to the place, and bone wax was used to fix the bone in place. The skin incision was closed with 6-0 suture and analgesia ( $2 \mathrm{mg} / \mathrm{kg}$ ketoprofen; intraperitoneal [IP]) was administered at the end of the surgery. The food intake and the body mass of the mice were measured every 48 hours until the euthanizing of animals.

\section{Endocytosis of RhoB/MTX-LNC on glial cells}

For assessment of MTX-LNC/RB endocytosis, GL261 and BV2 (20,000 cells) were seeded in sterile coverslips $(13 \mathrm{~mm}) 24$ hours before experiments. After adhesion, cells remained in the serum-deprived medium overnight. After that, cells were washed with PBS and incubated at different times $\left(1,5,20,40\right.$, or 60 minutes at $\left.37^{\circ} \mathrm{C}\right)$ with Hank's balanced salt solution (HBSS) containing MTX-LNC/RB $(5,500 \mathrm{nmol} / \mathrm{L})$. Then, cells were washed $(3 \times 5$ minutes $)$ with PBS and incubated ( 5 minutes, $37^{\circ} \mathrm{C}$ ) with HBSS containing 4', 6-diamidino-2-phenylindole (DAPI; 1:50) for cell nuclear localization. Cells were washed $(3 \times 5$ minutes) and fluorescent images were acquired by using a fluorescence microscope (AxioVision; Carl-Zeiss). The images were analyzed by using Zen 2012 (Carl-Zeiss) and the fluorescence intensity (arbitrary units; au) was measured in the region of interest by selecting just inside the cell. All the quantifications were carried out on backgroundsubtracted images. For each experiment, five field images that were randomly selected were analyzed, and between 6 and 8 cells were analyzed in each field. The results are shown as the percentage of RhoB/MTX-LNC endocytosis in comparison with control cells that were incubated with HBSS without RhoB/MTX-LNC. These experiments were conducted in order to determine whether RhoB/MTX-LNC are able to enter cells and the time necessary to maximal endocytosis of RhoB/MTX-LNC. In order to determine the concentration of endocytosis inhibitors, BV2 cells were seeded in sterile coverslips and incubated $\left(1\right.$ hour at $\left.37^{\circ} \mathrm{C}\right)$ with HBSS containing fluorescent probes used as positive control to phagocytosis (zymozan conjugated to Alexa Fluor $488-500 \mu \mathrm{g} / \mathrm{mL}$ ); or clathrin-mediated endocytosis (low-density lipoprotein [LDL] conjugated to Alexa Fluor $488-20 \mu \mathrm{g} / \mathrm{mL}$ ); or caveolae-mediated endocytosis (choleric toxin conjugated to Alexa Fluor 488-10 $\mu \mathrm{g} / \mathrm{mL}$ ). The pre-incubation of BV2 cells with Cyto D $(10 \mu \mathrm{mol} / \mathrm{L})$, Suc $(0.5 \mathrm{~mol} / \mathrm{L})$, or $\mathrm{M} \beta C(10 \mu \mathrm{mol} / \mathrm{L})$ was able to induce a significant decrease $(P<0.05)$ in the capitation of positive controls to phagocytosis, clathrin-mediated endocytosis, and caveolae-mediated endocytosis, respectively. 
A
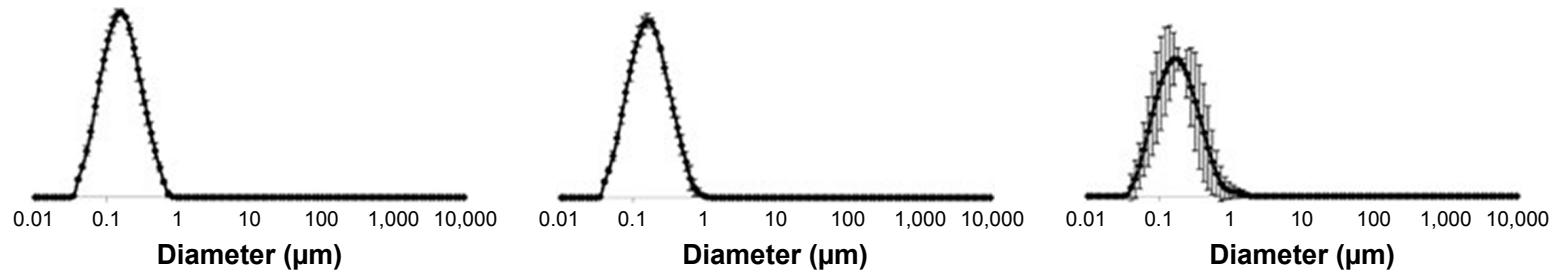

B
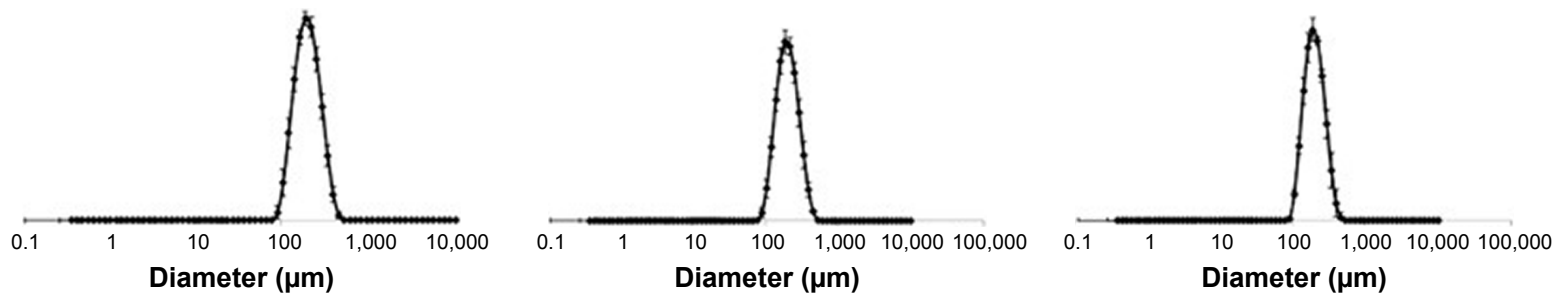

C
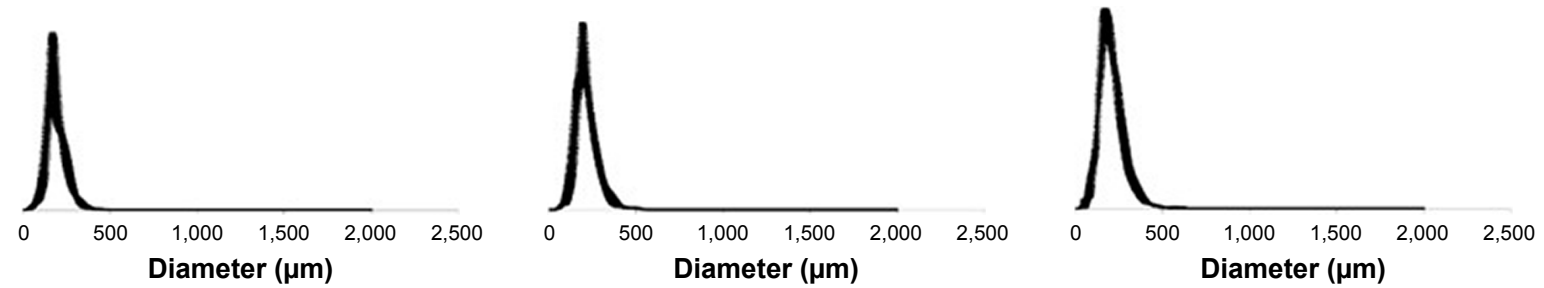

Figure SI (A) Particle size distributions obtained by laser diffraction (Mastersize 2000; Malvern Panalytical, Malvern UK); (B) dynamic light scattering (Zetasizer ZS; Malvern Panalytical, Malvern, UK); and (C) nanoparticle tracking analysis (Nanosight LMI0; Nanosight, Malvern Panalytical, Malvern, UK) for LNC (left), MTX-LNC (center) and rhodamine B-labeled LNC-MTX (right). Mean \pm SD for triplicate batches $(n=3)$.

Abbreviations: LNC, lipid-core nanocapsules; MTX-LNC, methotrexate-loaded lipid-core nanocapsules.

A

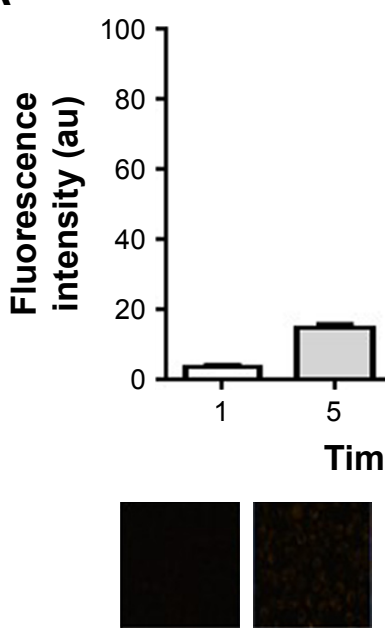

B

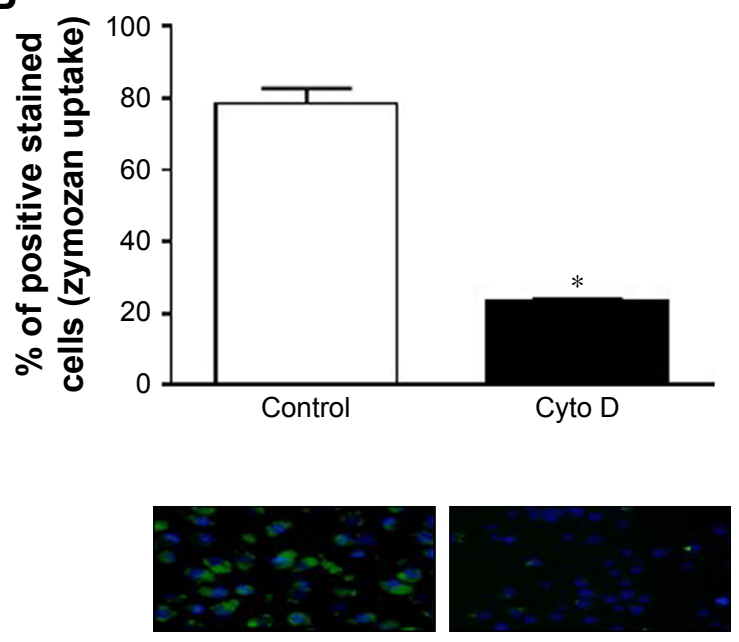

Figure S2 (Continued) 
C

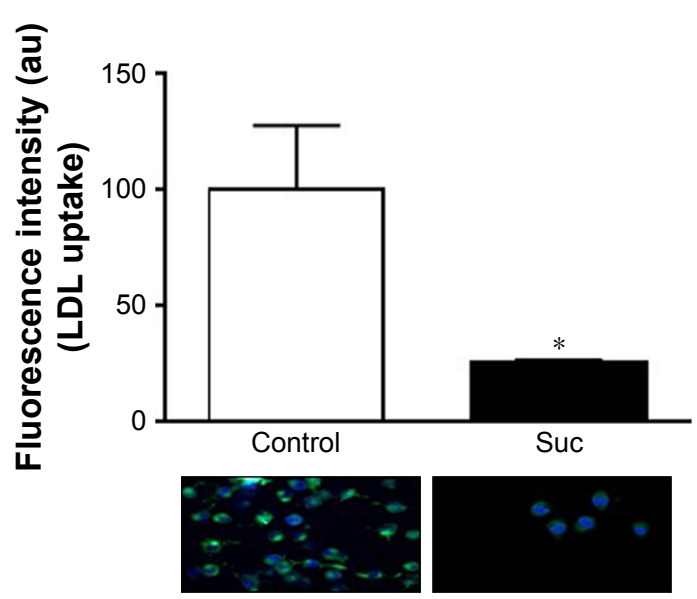

D

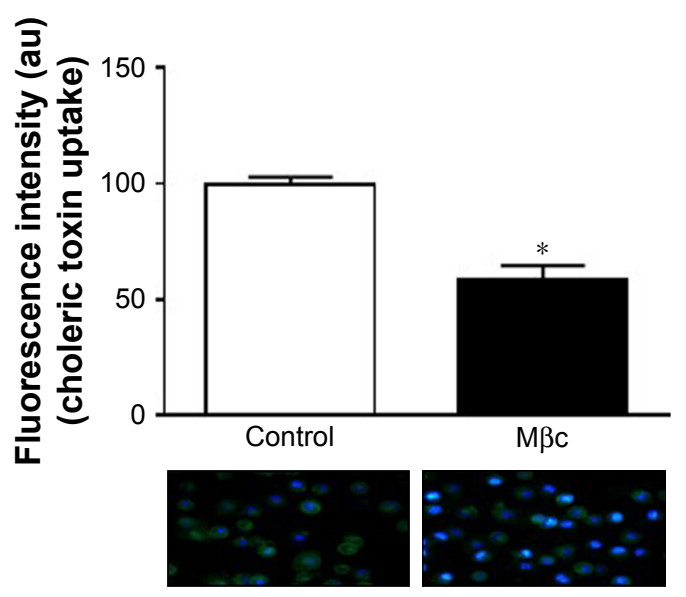

Figure S2 (A) Bar graphs and representative images showing fluorescence of RhoB/MTX-LNC (5,500 nmol/L) in BV2 cells after I, 5, 20, 40, or 60 minutes of incubation. (B) Positive control of phagocytosis-mediated endocytosis. BV2 cells were incubated with HBSS containing zymozan conjugated to Alexa Fluor 488 (500 $\mu g / m L)$ in the absence (control) or presence of Cyto D (10 $\mu \mathrm{mol} / \mathrm{L})$. (C) Positive control of clathrin-mediated endocytosis. BV2 cells were incubated with HBSS containing LDL conjugated to Alexa Fluor $488(20 \mu \mathrm{g} / \mathrm{mL})$ in the absence (control) or presence of high concentration of Suc ( $0.5 \mathrm{~mol} / \mathrm{L})$. (D) Positive control of caveolin-mediated endocytosis. BV2 cells were incubated with HBSS containing choleric toxin conjugated to Alexa Fluor $488(10 \mu \mathrm{g} / \mathrm{mL}$ ) in the absence (control) or presence of $\mathrm{M} \beta \mathrm{C}$ ( $5 \mathrm{mmol} / \mathrm{L})$. For phagocytosis analyses, the number of labeled cells (presence of phagosome) was counted, whereas for clathrin- or caveolin-mediated endocytosis, the fluorescence intensity (au) within cells was determined. In all the cases, the control group was considered as the $100 \%$ reference. The results are expressed as mean \pm SEM, $n=3-5$ per group. Data from graph (A) were analyzed by one-way ANOVA, whereas (B-D) were analyzed by the Student's $t$-test, $* P<0.05$ vs control.

Abbreviations: LDL, low-density lipoprotein; HBSS, Hank's balanced salt solution; SEM, standard error of the mean; ANOVA, analysis of variance.

Sham

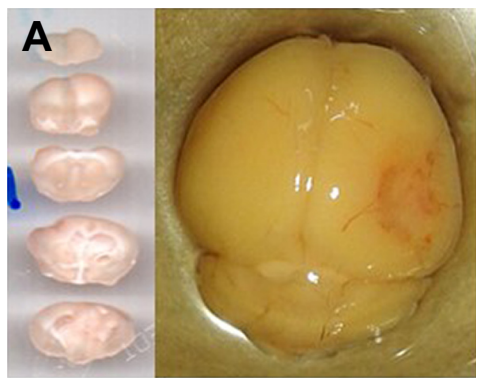

Saline

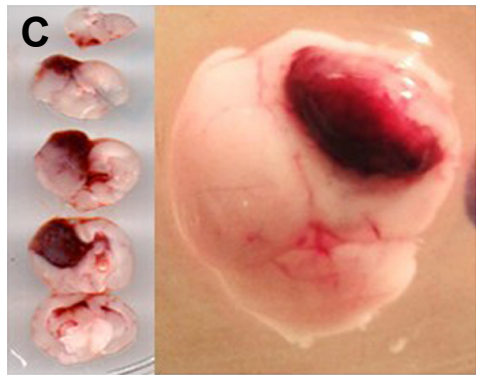

MTX

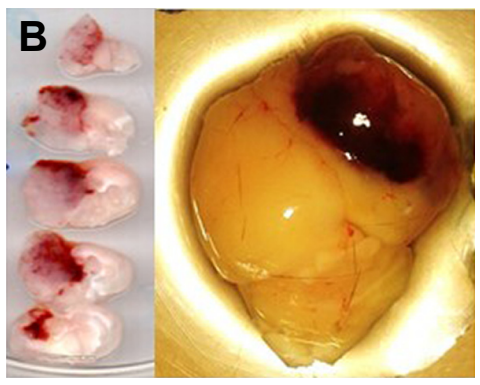

MTX-LNC

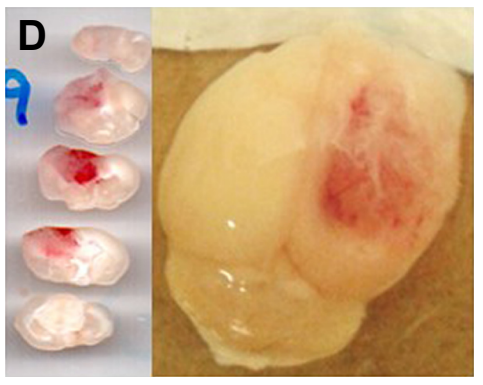

Figure S3 Representative images of mice brain segments: (A) sham-group; glioblastoma-carrying mice treated with MTX solution (B), saline (C), or MTX-LNC (D) for 10 days, in alternated days, by the oral route.

Abbreviations: MTX, methotrexate; MTX-LNC, methotrexate-loaded lipid-core nanocapsules.

International Journal of Nanomedicine

Dovepress

\section{Publish your work in this journal}

The International Journal of Nanomedicine is an international, peerreviewed journal focusing on the application of nanotechnology in diagnostics, therapeutics, and drug delivery systems throughou the biomedical field. This journal is indexed on PubMed Central, MedLine, CAS, SciSearch ${ }^{\circledR}$, Current Contents ${ }^{\circledR} /$ Clinical Medicine,
Journal Citation Reports/Science Edition, EMBase, Scopus and the Elsevier Bibliographic databases. The manuscript management system is completely online and includes a very quick and fair peer-review system, which is all easy to use. Visit http://www.dovepress.com/ testimonials.php to read real quotes from published authors.

\footnotetext{
Submit your manuscript here: http://www.dovepress.com/international-journal-of-nanomedicine-journal
} 